\title{
Describing temporal variation in reticuloruminal pH using continuous monitoring data
}

\author{
M. J. Denwood, ${ }^{\star 1}$ J. L. Kleen,† D. B. Jensen, ${ }^{\star}$ and N. N. Jonsson $¥ \S$ \\ ${ }^{*}$ Department of Veterinary and Animal Sciences, University of Copenhagen, 1870 Frederiksberg C, Denmark \\ †CowConsult, Coldinne, 26532, Germany \\ łInstitute of Biodiversity Animal Health and Comparative Medicine, University of Glasgow, Glasgow, G61 1QH, United Kingdom \\ §Harbro Ltd., Birkhill Mill, Lanarkshire, ML11 ONJ, United Kingdom
}

\section{ABSTRACT}

Reticuloruminal $\mathrm{pH}$ has been linked to subclinical disease in dairy cattle, leading to considerable interest in identifying $\mathrm{pH}$ observations below a given threshold. The relatively recent availability of continuously monitored data from $\mathrm{pH}$ boluses gives new opportunities for characterizing the normal patterns of $\mathrm{pH}$ over time and distinguishing these from abnormal patterns using more sensitive and specific methods than simple thresholds. We fitted a series of statistical models to continuously monitored data from 93 animals on 13 farms to characterize normal variation within and between animals. We used a subset of the data to relate deviations from the normal pattern to the productivity of 24 dairy cows from a single herd. Our findings show substantial variation in $\mathrm{pH}$ characteristics between animals, although animals within the same farm tended to show more consistent patterns. There was strong evidence for a predictable diurnal variation in all animals, and up to $70 \%$ of the observed variation in $\mathrm{pH}$ could be explained using a simple statistical model. For the 24 animals with available production information, there was also a strong association between productivity (as measured by both milk yield and dry matter intake) and deviations from the expected diurnal pattern of $\mathrm{pH} 2 \mathrm{~d}$ before the productivity observation. In contrast, there was no association between productivity and the occurrence of observations below a threshold $\mathrm{pH}$. We conclude that statistical models can be used to account for a substantial proportion of the observed variability in $\mathrm{pH}$ and that future work with continuously monitored $\mathrm{pH}$ data should focus on deviations from a predictable pattern rather than the frequency of observations below an arbitrary $\mathrm{pH}$ threshold.

Received March 3, 2017

Accepted August 27, 2017.

${ }^{1}$ Corresponding author: md@sund.ku.dk
Key words: reticuloruminal $\mathrm{pH}$, acidosis, remote sensing data, statistical model

\section{INTRODUCTION}

Assessment of reticuloruminal $\mathrm{pH}$ in cattle has been used as an indicator of an excessive intake of soluble carbohydrate or a shortage of physically effective fiber and the consequent predisposition to a range of health problems, including ruminitis, liver abscess, left displaced abomasum, diarrhea, laminitis, and poor milk production (Dirksen and Smith, 1987; Nordlund and Garrett, 1997; Garrett et al., 1999; Plaizier et al., 2008). Evaluation of reticuloruminal $\mathrm{pH}$ is therefore of interest, with most clinicians using a single observation to define the status (Nordlund et al., 1995; Nordlund and Garrett, 1997). It is now possible to continuously monitor $\mathrm{pH}$ values using remote sensing data, but this generates a large volume of data that can be very challenging to interpret. As a result, researchers using continuous $\mathrm{pH}$ monitoring techniques to investigate diet and acidosis have most often used average values or threshold approaches for evaluating reticuloruminal pH. For example, Khafipour et al. (2011) used the overall mean $\mathrm{pH}$ for an individual animal over a period of time and the number of minutes that the $\mathrm{pH}$ was seen to be below 5.6. We aim to define and diagnose abnormal reticuloruminal $\mathrm{pH}$ by identifying deviations from normal $\mathrm{pH}$ patterns rather than the number of observations below a given threshold. To achieve this goal, we first require the range of normal $\mathrm{pH}$ conditions to be described and quantified.

Statistical methods can be used to help quantify and explain some of the observed variability in $\mathrm{pH}$ over time and may also be able to identify periods of the $\mathrm{pH}$ time series that are not broadly consistent with predictions. These discrepancies can be quantified based on residual variation between the fitted model and observations. Several statistical methods can be used to deal with time series data with temporal patterns, including re- 
curring temporal patterns with known frequency, such as predictable daily fluctuations. A descriptive method (e.g., a linear decomposition or smoothed average) can be used to separate the predictable temporal variation from the longer term patterns in the data. For example, Maatje et al. (1992) modeled the daily milk yield of dairy cows, which is known to follow a characteristic curve over time (Wood, 1967; Cole et al., 2009), using a simple moving average in which significant deviations between the observed milk yield and the yield predicted by the moving average were considered to be indicative of mastitis. More recently, Ostersen et al. (2010) implemented an estrus detection model based on the diurnal frequency of individual sows visiting a boar by dividing the $24 \mathrm{~h}$ of the day into 4 periods of $6 \mathrm{~h}$ and then assigning an expected average frequency per period.

Another method is to fit a model that includes sinusoidal effects (with known frequency and varying amplitude and phase shift) to account for and analyze the recurring patterns. Fitting increasing numbers of these sine waves with different frequencies allows any temporally recurring pattern to be described using the sum of its harmonics, although at the cost of a potentially large number of degrees of freedom. For example, Madsen et al. (2005) modeled the daily drinking pattern of weaned pigs using between 1 and 12 harmonic waves implemented within a dynamic linear model (DLM). The same authors have also shown that significant deviations between this model and the observed drinking pattern correlate with undesirable events such as diarrhea and herd management problems (Madsen and Kristensen, 2005). Similarly, Jensen et al. (2017) modeled the drinking behavior of slaughter pigs using the sum of 3 harmonic waves implemented in a DLM with the purpose of detecting diarrhea or pen fouling.

A third option is to fit 1 or more sine waves at frequencies corresponding to known (or suspected) biologically relevant influences on the parameter of interest. For example, a sine wave with a frequency corresponding to a daily cycle over $24 \mathrm{~h}$ could be an appropriate way to deal with predictable daily variation due to a regular routine. We prefer this option because it allows the most intuitive interpretation in terms of biologically relevant factors and allows for extrapolation to other herds and animals.

In this paper, we make use of continuously monitored reticuloruminal $\mathrm{pH}$ values obtained from clinically normal dairy cattle. We first quantify the variation in $\mathrm{pH}$ observed within and between animals and farms using standard linear models and then more closely examine the subtle differences in $\mathrm{pH}$ patterns using more complex nonlinear models applied to the data from each animal separately. The remaining residual variation is then used to identify periods of abnormality that can be related to decreased production traits even in these clinically normal cattle. The overall aim is to produce recommendations and statistical methods for distinguishing "normal" from "abnormal" when dealing with long-term continuously monitored $\mathrm{pH}$ data.

\section{MATERIALS AND METHODS}

\section{Rumen Bolus Data}

Remotely sensed pH data were obtained from 13 different dairy farms in Germany, with a total of 93 animals. Feeding management was consistent within each herd over the course of the data collection, and there were no reports or suspicions of either clinical disease or abnormal rumen function in any of the animals. The dairy farms were divided into those milking 3 times per day (group A: 8 farms with a total of 45 animals) and those milking twice per day (group B: 5 farms with a total of 48 animals). The 93 data series (45 from group A and 48 from group B) were first analyzed together to quantify the importance of the variation between herds and animals and then analyzed separately to allow the difference in $\mathrm{pH}$ patterns between individuals to be explored in more detail. An overview of the complete procedure is given in Figure 1.

Wireless, indwelling rumen $\mathrm{pH}$ and temperaturemonitoring boluses with a measurement interval of $600 \mathrm{~s}$ were used in all animals (smaXtec Animal Care GmbH, Graz, Austria). The pH probes of the boluses were all calibrated in $\mathrm{pH} 4$ and $\mathrm{pH} 7$ buffer solutions before administration to the animals by mouth. Individual $\mathrm{pH}$ records were graphically assessed to remove any readings that were not compatible with rumen conditions ( $\mathrm{pH} \geq 10$ or constant $\mathrm{pH}$ ) from the beginning and end of the time series. Additionally, the first $12 \mathrm{~h}$ and the last hour were removed from all data series to allow calibration time and eliminate unreliable readings from failing boluses, respectively. All data series were limited to a maximum of $50 \mathrm{~d}$ of observation per the manufacturer's guarantee.

\section{Summary Statistics}

Crude summary statistics of mean, variance, first percentile, and 99th percentile were calculated for the $\mathrm{pH}$ data series from each animal. The effect of farm on the 93 recorded values of these 4 within-animal summary statistics was quantified using 4 separate linear models in R (R Core Team, 2016), with farm as a fixed effect. 
Table 1

$\rightarrow$ Figure 2

13 Farms, 93 Animals

Figure 3

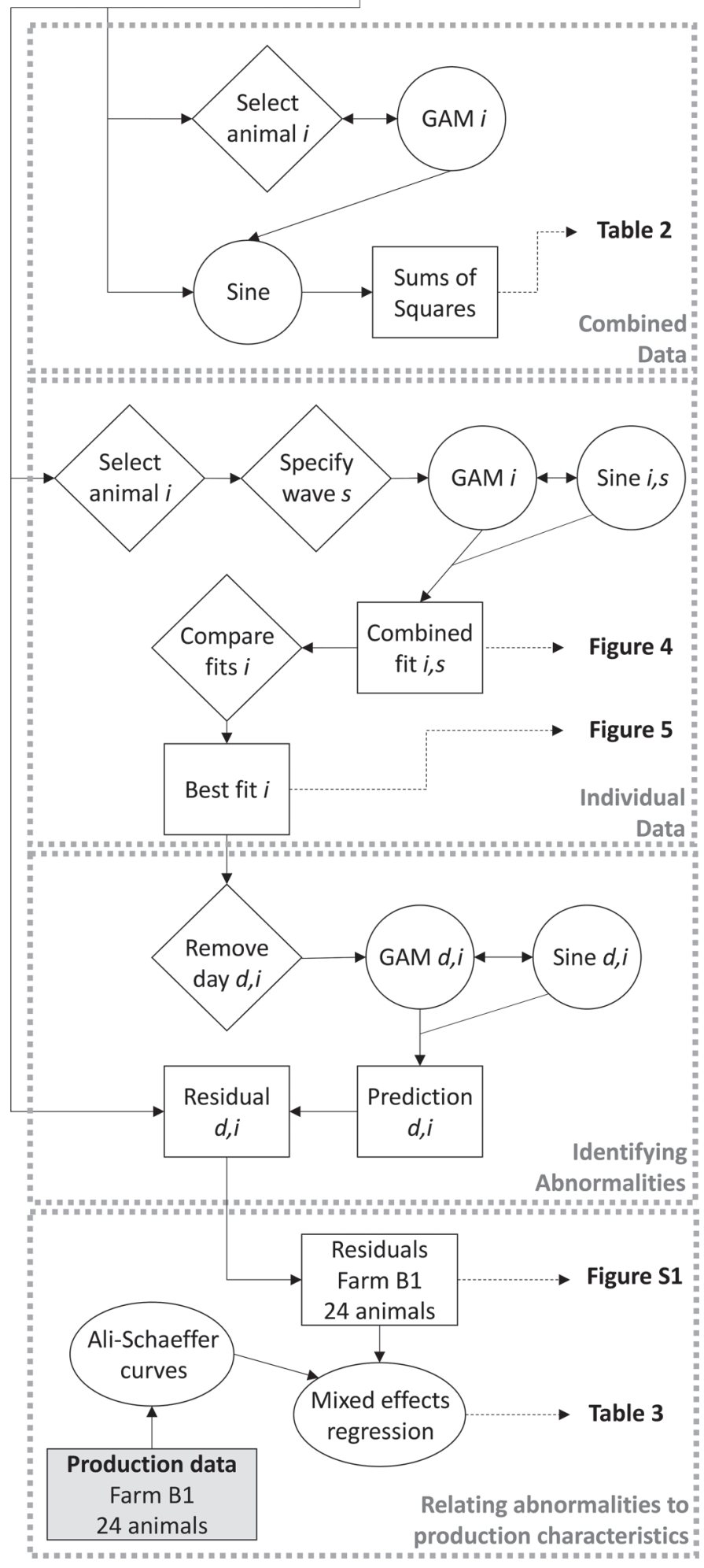

Figure 1. A flow chart representation of the complete analysis procedure as used for this article, with reference to the sections given in Materials and Methods. GAM $=$ generalized additive model.

\section{Statistical Methods for Combined Data}

The statistical models used to analyze the data were based on sinusoidal functions. The standard linear formulation of a sine wave is given by

$$
A \sin \left(\theta+\theta_{0}\right)=\alpha \sin \theta+\beta \cos \theta,
$$

where $\theta$ is a known parameter reflecting the time of day that is transformed to the interval $(0,2 \pi), A$ is the amplitude, and $\theta_{0}$ is the phase shift. The 2 terms $\alpha$ and $\beta$ are linear parameters on the interval $(-\infty, \infty)$ that are typically estimated and are related to $A$ and $\theta_{0}$ by

$$
A=\sqrt{\alpha^{2}+\beta^{2}} ; \theta_{0}=\operatorname{atan} 2(\beta, \alpha),
$$

where atan2 is the 2-argument arc tangent.

A fixed frequency of 1 cycle per day was used for the first sine wave to describe diurnal variation. In addition, a second sine wave with a fixed frequency corresponding to 2 or 3 cycles per day (but with equal spacing between the times of milking events rather than equal spacing throughout the day) may be justified to explain the influence of farm management factors associated with the frequency of daily milking. The $\theta$ parameter was therefore derived from the time of day to correspond to 2 different hypothesized cyclical events: diurnal variation on a 24-h cycle and cyclical patterns corresponding to each milking event. The total within-day variation was modeled as the sum of up to 2 separate sine waves that were simultaneously fitted using periods of (1) 24 $\mathrm{h}$ and (2) 2 or 3 daily milking events (depending on the on-farm milking frequency). The daily milking events were timed to correspond to unequally spaced milking times, which were approximated for all farms as 0430 and $1530 \mathrm{~h}$ for twice-daily milking and 0430, 1300, and $2000 \mathrm{~h}$ for thrice-daily milking.

In addition to the cyclical temporal patterns discussed above, there may be longer term temporal trends that can affect the average $\mathrm{pH}$ on this longer time scale. Therefore, before fitting the linear model, a generalized additive model (GAM) was used to account for the longer term changes in $\mathrm{pH}$ between days within each individual animal. A separate GAM was fitted to the $\mathrm{pH}$ data series from each animal using the mgcv package (Wood, 2011) for R (R Core Team, 2016), and the estimated spline functions were extracted and used to generate offset covariates for the linear model for each animal.

The complete set of observed $\mathrm{pH}$ data (616,945 observations from 93 animals) was then fitted to the linear model with 2 sinusoidal functions using offset covariates obtained from the GAM. Each model incorporated 
linear parameters of $\alpha_{1}$ and $\beta_{1}$ for the daily sine wave, $\alpha_{2}$ and $\beta_{2}$ for the milking-frequency sine wave, and fixed effects of farm and animal. The equation for the main effects of this model is given as

$$
\begin{aligned}
\mathrm{pH}= & {\left[c+m_{f}+n_{a}+\alpha_{1} \sin \theta_{\mathrm{D}}+\beta_{1} \cos \theta_{\mathrm{D}}\right.} \\
& \left.+\alpha_{2} \sin \theta_{\mathrm{M}}+\beta_{2} \cos \theta_{\mathrm{M}}\right]+\left[s_{a}(t)\right],
\end{aligned}
$$

where $c$ is the intercept, $m_{f}$ is the main effect associated with the relevant farm, $n_{a}$ is the main effect associated with the relevant animal, $\theta_{\mathrm{D}}$ represents the time of day, $\theta_{\mathrm{M}}$ represents the time elapsed since the last milking event, $s_{a}$ is a smoothed spline function for animal $a$, and $t$ is the time elapsed since the start of the $\mathrm{pH}$ series for that animal (date and time). The 2 sets of square brackets denote the elements of the model fitted using the linear model (left) and GAM (right). The models were fitted using $\mathrm{R}$ ( $\mathrm{R}$ Core Team, 2016), including all 4 interactions between $m_{f}$ and the $\alpha_{1}, \beta_{1}, \alpha_{2}$, and $\beta_{2}$ parameters as well as all 4 interactions between $n_{a}$ and the $\alpha_{1}, \beta_{1}, \alpha_{2}$, and $\beta_{2}$ parameters.

\section{Statistical Methods for Individual Animal Data}

A more complex modeling procedure was then used to further examine the nature of any differences in $\mathrm{pH}$ patterns between animals. These models were fitted to the data series from each animal separately so that the estimated patterns obtained from each animal were not influenced by the data from other animals.

The standard sine wave model was extended to allow the value of the 2 rates of change to vary so that the increase in $\mathrm{pH}$ from the minimum to maximum value might occur more quickly than the decrease in $\mathrm{pH}$ from the maximum to minimum value. Such extensions to the standard sine wave are not possible using a linear model system but can be made using a nonlinear model system. A nonlinear periodic function was therefore written to extend the standard sine wave model with an additional parameter to allow the shape of the wave to be skewed so that the minimum trough points could be moved relative to their standard equidistant position between maximum peaks. This mechanism allows a skew parameter, $\gamma$, to control the rate of change for increasing and decreasing $\mathrm{pH}$ phases while still retaining the amplitude, periodicity, and phase of the standard sine wave model. This parameter is also defined on the interval $(-\infty, \infty)$ to facilitate estimation, although it is subsequently transformed to the interval $(-0.5,0.5)$ using a scaled and recentered logistic transformation. The final skewed sine wave is therefore a nonlinear function of $\theta$ (which is derived from the time of day, as discussed below) and the 3 parameters $\alpha, \beta$, and $\gamma$. The $\mathrm{R}$ code that implements this nonlinear function is given as an online supplement (Supplemental Material; https://doi .org/10.3168/jds.2017-12828), and examples of the output of this function for a small number of possible values for $\gamma$ are shown in Figure 2. Note that the standard sine wave is a special case of this function with skew parameter $\gamma=0$, which was also used as a baseline model to test the improvement in model fit achieved by allowing $\gamma$ to vary. The 3 parameters $\alpha, \beta$, and $\gamma$ of the nonlinear function were optimized simultaneously using the nlsLM function in the minpack.lm package (Elzhov et al., 2013) for R (R Core Team, 2016).

As with the simpler models described above, 2 separate sine waves with frequencies of 1 oscillation per 24 $\mathrm{h}$ and either 2 or 3 oscillations per $24 \mathrm{~h}$ were used to explain diurnal variation and the influence of farm management factors associated with the frequency of daily milking. A GAM was also used to account for longer term temporal trends within each animal as before, although the GAM and nonlinear components were fitted iteratively (each using the estimates from the other model as fixed covariates) for this more complex model to allow the combined likelihood to be maximized. The complete model for each animal is represented by

$$
\begin{aligned}
& \mathrm{pH}=\left[c+\mathrm{f}\left(\theta_{D}, \alpha_{1}, \beta_{1}, \gamma_{1}\right)\right. \\
& \left.+\mathrm{f}\left(\theta_{M}, \alpha_{2}, \beta_{2}, \gamma_{2}\right)\right]+[\mathrm{s}(t)],
\end{aligned}
$$

where the function $\mathrm{f}$ is the skewed sine function, $\mathrm{s}$ is a smoothed spline function, $\theta_{D}$ represents the time of day, $\theta_{M}$ represents the time elapsed since the last milking event, and $t$ is the time elapsed since the start of the $\mathrm{pH}$ series for that animal (date and time). The 2 sets of square brackets denote the nonlinear (left) and GAM (right) elements of the model.

Model fit for the more complex models was compared in 2 distinct ways. First, Akaike's information criterion (AIC; Akaike, 1973) was compared between the models, with the total number of degrees of freedom equal to the number of parameters in the nonlinear model plus the effective number of parameters used by the GAM. The motivation for this was to assess the improvement in statistical fit obtained from increasing the complexity of the model for each animal. Second, the incremental reduction in residual variance was evaluated from each successively more complex model. The motivation for this was to assess the biological effect of the more complex relationships in the model in terms of the variance explained. To simplify model comparisons, the skew parameter was also included in the milkingrelated sine wave function if including the same parameter in the daily sine wave function improved the AIC of the model. An additional comparison was made 
between the 4 different possibilities for milking-related sine waves (2 and 3 times daily, each spaced equally or corresponding to approximate milking times), which had equal degrees of freedom permitting a direct comparison of the maximized likelihoods.

\section{Identifying Abnormalities in $\mathrm{pH}$}

The magnitude of the residuals (difference between the $\mathrm{pH}$ values predicted by a fitted model and those observed from the data) can be used to identify periods during which abnormalities have occurred. Residuals can be calculated as either signed or absolute, with the former taking into account the difference between posi-

a)

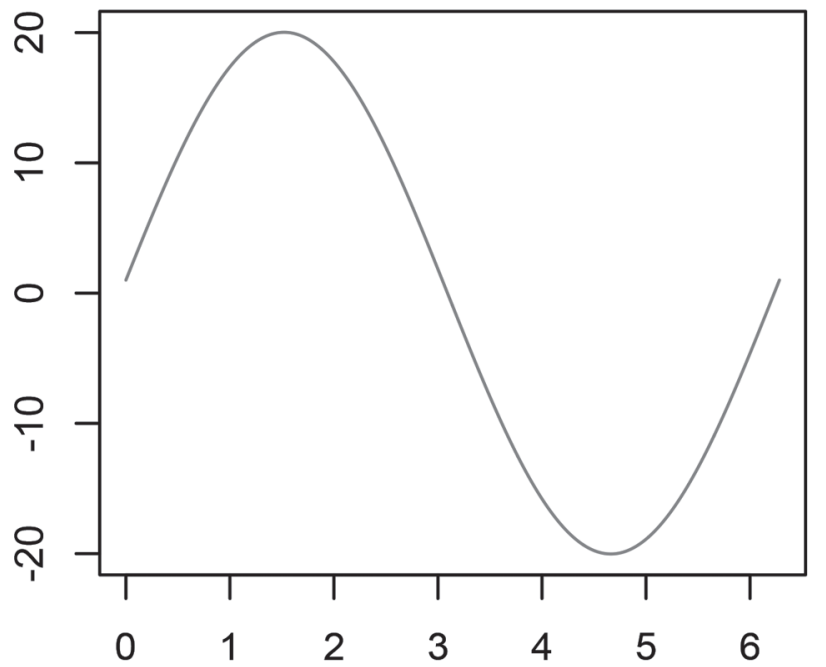

c)

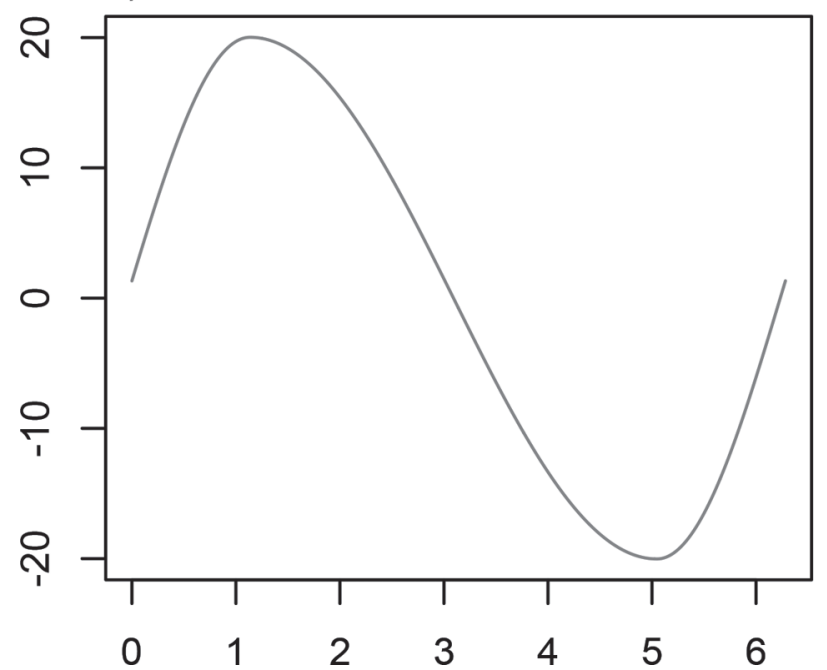

tive residuals (underestimates) and negative residuals (overestimates) and the latter removing the sign so that only the magnitude is recorded. We summarized these residuals over 24 -h periods so that the degree of predictability or abnormality was quantified for each animal and observed day. Rather than basing the predictions on the entire data series, the model from which predictions were made was sequentially refitted to a subset of the $\mathrm{pH}$ data for each observed day. This data subset excluded the 3-d period of observations from the day before to the day following that for which predictions and residuals were to be calculated, thus avoiding any potential bias from including the same data in calculating both the model fit and residuals. Each animal was

\section{b)}

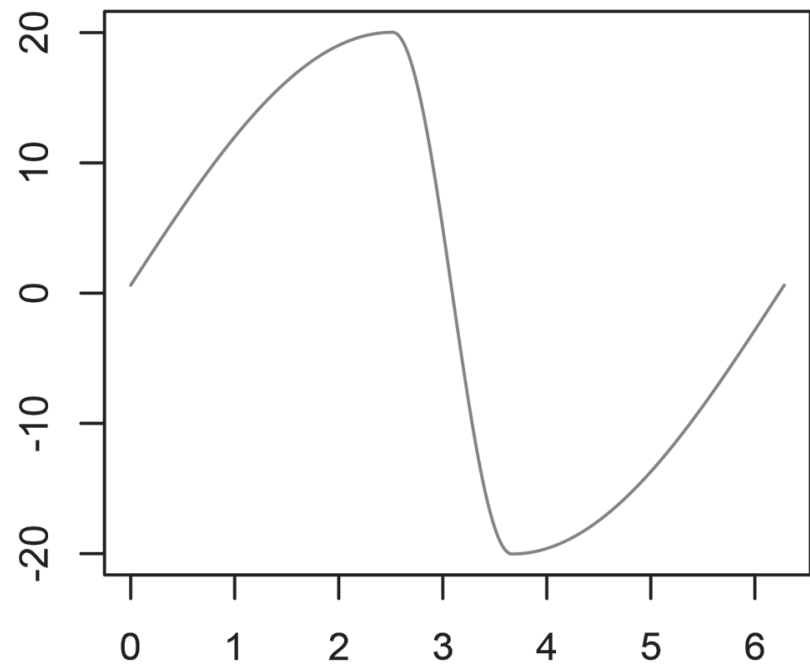

d)

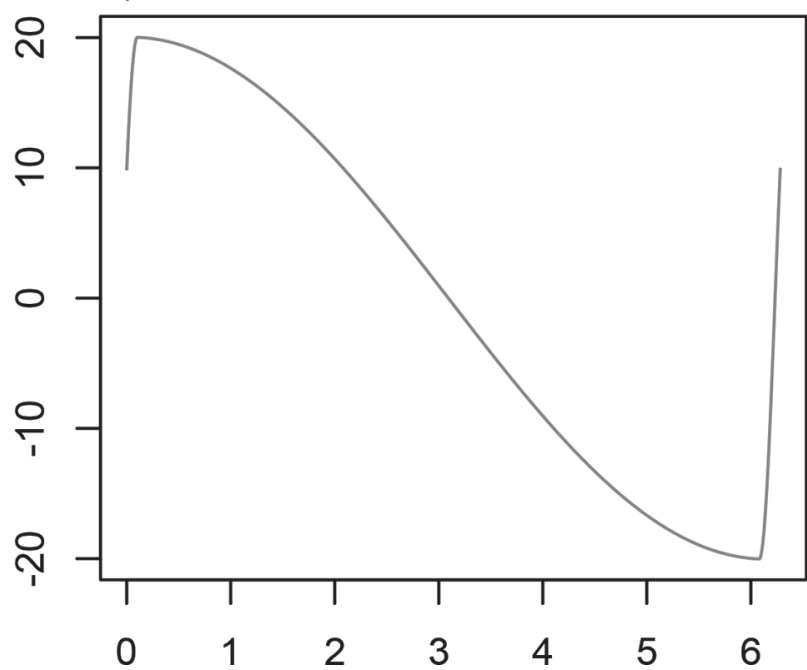

Figure 2. Examples of sine waves for $\theta$ in the interval $(0,2 \pi)$ corresponding to the complete range of values in, for example, time of day. The skew parameter $\gamma$ is shown varying in the set (a) $0,(\mathrm{~b}) 1.5,(\mathrm{c})-0.5$, and (d) -3 with constant phase shift $\left(\theta_{0}=0\right)$ and amplitude $(A=$ 20) parameters. Note that the standard sine wave is a special case of $\gamma=0$. 
modeled separately using the best-fitting model for the separate data, as described above.

Residuals from each separately fitted model were calculated for the relevant day, and the results were combined into a single series of residuals for each observation per animal. These were subsequently summarized for each 24-h time period into (1) the mean of the residuals for the day and (2) the mean of the absolute residuals for the day, which respectively represent (1) a period of unexpected sustained acidosis or alkalosis and (2) a period of unpredictability in $\mathrm{pH}$ (regardless of direction). Summary statistics based on the raw data were also used, representing the number of $\mathrm{pH}$ observations for that day that were greater than an animalspecific upper threshold and the number of $\mathrm{pH}$ observations that day that were lower than an animal-specific lower threshold. These thresholds were taken from the first and 99th percentiles (i.e., $1 \%$ most acidic and 1\% most alkaline) over the entire observation period for that animal, as previously calculated. These summary statistics were intended to be closer in concept to the traditional $\mathrm{pH}$ threshold for declaring acidosis, with the exception of using an animal-dependent threshold.

\section{Relating Abnormalities to Production Characteristics}

To demonstrate the ability of our statistical methods to identify abnormalities in $\mathrm{pH}$ series, we related the 4 daily summary statistics given above to contemporary daily measurements of DMI and milk yield available for the 24 animals in herd B1. To simplify the comparisons, the Ali-Schaeffer curve (Ali and Schaeffer, 1987; Buttchereit et al., 2010) was used to standardize the observed daily milk yields by days since the start of the lactation. Multivariate linear mixed effects regression models were used to model the daily productivity of all 24 cows using the cow ID as a random effect and fixed effects relating to the 4 summary statistics of $\mathrm{pH}$ measurements and model predictions, as given above. Separate models were fitted to the 2 productivity parameters of DMI recordings and standardized milk yields using identical predictor variables. The data were aligned so that summary statistics for $\mathrm{pH}$ measurements and model predictions were used from $2 \mathrm{~d}$ before the corresponding productivity observations to maximize the potential predictive ability of the model by using the smallest time lag that would be useful in practice. All linear mixed effects regression models were fitted using the lme4 package (Bates et al., 2014) for R (R Core Team, 2016).

\section{RESULTS}

\section{Summary Statistics}

The number and length of $\mathrm{pH}$ series obtained are shown in Table 1, and the mean and variance of individual animal $\mathrm{pH}$ series are shown in Figure 3. There is a substantial variation in the individual animal characteristics of $\mathrm{pH}$, although there was also a significant effect of farm on the mean $\left(P<0.001\right.$, adjusted $\mathrm{R}^{2}$ $=0.32)$, variance $\left(P<0.001\right.$, adjusted $\left.\mathrm{R}^{2}=0.44\right)$, first percentile $\left(P=0.002\right.$, adjusted $\left.\mathrm{R}^{2}=0.20\right)$, and 99th percentile $\left(P<0.001\right.$, adjusted $\left.\mathrm{R}^{2}=0.37\right)$ of the observed $\mathrm{pH}$ measurements for each animal.

\section{Combined Data Results}

Modeling results from the combined data set are shown in Table 2 . The mean squares estimates indicate that both daily and milking-frequency sine waves are important components of $\mathrm{pH}$ variation and that mean

Table 1. The number of $\mathrm{pH}$ series per farm, mean (range) number of $\mathrm{pH}$ observations per animal, mean (range) length of time represented within the time series, and the number of time series for which Akaike's information criterion demonstrated the best model fit for a simple sine wave ( $2 \mathrm{df})$, sine wave with skew parameter ( $3 \mathrm{df})$, double sine wave with a second wave describing the known milking events (4 df), and both double sine wave and skew parameters $(6 \mathrm{df})$

\begin{tabular}{|c|c|c|c|c|c|c|c|}
\hline Farm & No. of animals & No. of observations & Days & Sine & Skew & Double & Both \\
\hline A1 & 5 & $7,125(7,120-7,129)$ & $49.5(49.5-49.5)$ & 0 & 0 & 2 & 3 \\
\hline $\mathrm{A} 2$ & 2 & $4,520(1,912-7,129)$ & $31.4(13.3-49.5)$ & 0 & 0 & 0 & 2 \\
\hline A3 & 6 & $6,812(6,528-7,099)$ & $47.5(45.5-49.5)$ & 0 & 0 & 1 & 5 \\
\hline A4 & 11 & $6,403(4,829-7,129)$ & $44.5(33.5-49.5)$ & 0 & 0 & 1 & 10 \\
\hline A5 & 3 & $7,107(7,081-7,121)$ & $49.5(49.5-49.5)$ & 0 & 0 & 1 & 2 \\
\hline A 6 & 5 & $5,267(4,743-5,460)$ & $36.6(33.0-37.9)$ & 0 & 0 & 0 & 5 \\
\hline A7 & 6 & $6,929(6,522-7,129)$ & $48.1(45.3-49.5)$ & 0 & 0 & 0 & 6 \\
\hline A 8 & 7 & $7,113(7,026-7,129)$ & $49.4(48.8-49.5)$ & 0 & 0 & 1 & 6 \\
\hline B1 & 24 & $6,598(6,595-6,600)$ & $45.8(45.8-45.8)$ & 0 & 0 & 0 & 24 \\
\hline B2 & 6 & $6,273(2,046-7,129)$ & $43.6(14.3-49.5)$ & 0 & 0 & 2 & 4 \\
\hline B3 & 4 & $7,044(6,792-7,129)$ & $48.9(47.2-49.5)$ & 0 & 0 & 1 & 3 \\
\hline B4 & 6 & $7,116(7,097-7,129)$ & $49.5(49.5-49.5)$ & 0 & 0 & 0 & 6 \\
\hline B5 & 8 & $6,886(6,643-7,122)$ & $48.0(46.4-49.5)$ & 1 & 0 & 1 & 6 \\
\hline
\end{tabular}


$\mathrm{pH}$ varies dramatically between farms (and to a lesser extent between animals on the same farm). The results also suggest that the precise pattern of $\mathrm{pH}$ variation varies to some extent between farms but appears to be more consistent between animals on the same farm. $F$-tests indicated that all fixed effect terms and interactions were significant $(P<0.001)$, although these $P$-values should be interpreted with caution due to the nonindependence of residuals in the fitted model.

\section{Individual Animal Data Results}

For the purposes of illustration, the 3 individual components of the more complex statistical model corresponding to the GAM, daily-frequency sine wave, and milking-frequency sine wave (as estimated from the data for 2 representative animals) can be seen in Figure 4. The proportion of the variance explained by the GAM component was between approximately 0 and $90 \%$ de-

a)

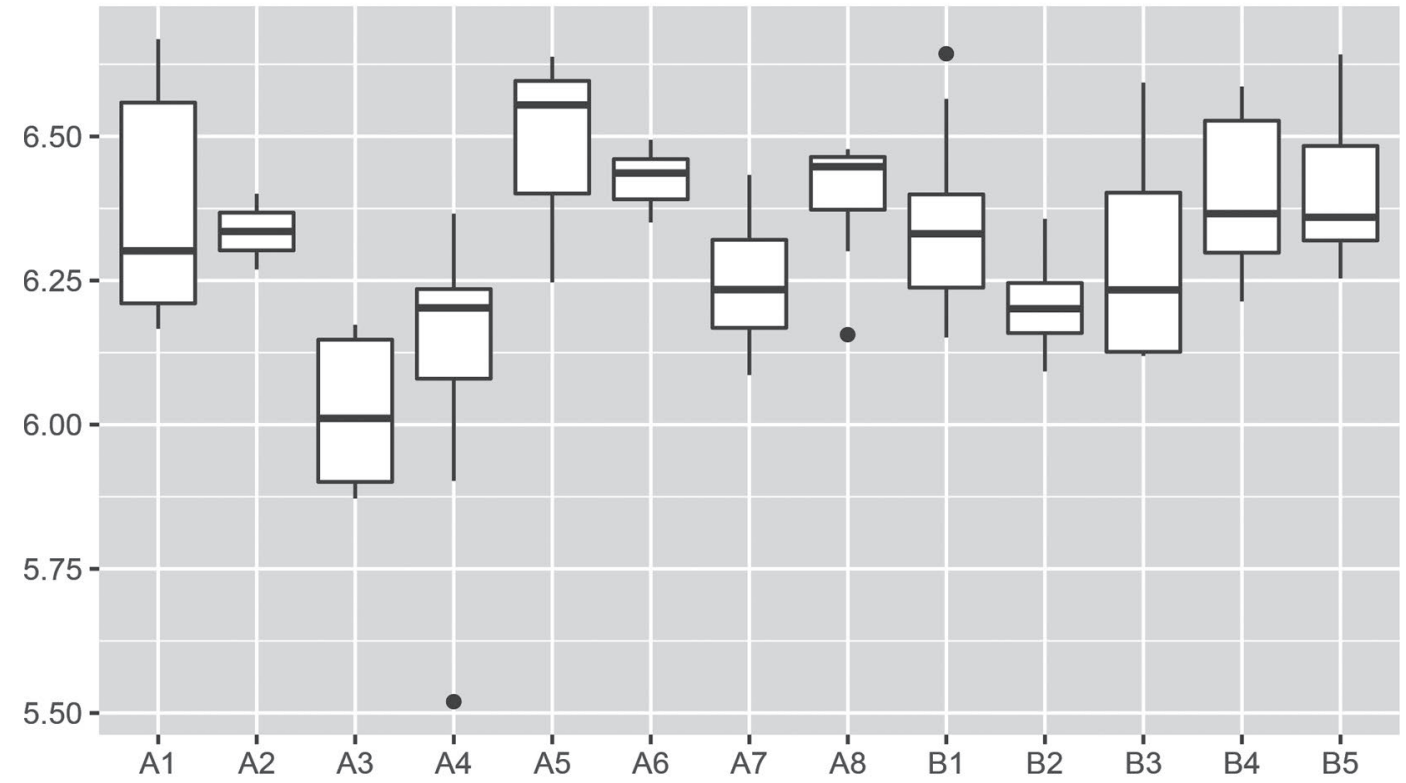

b)

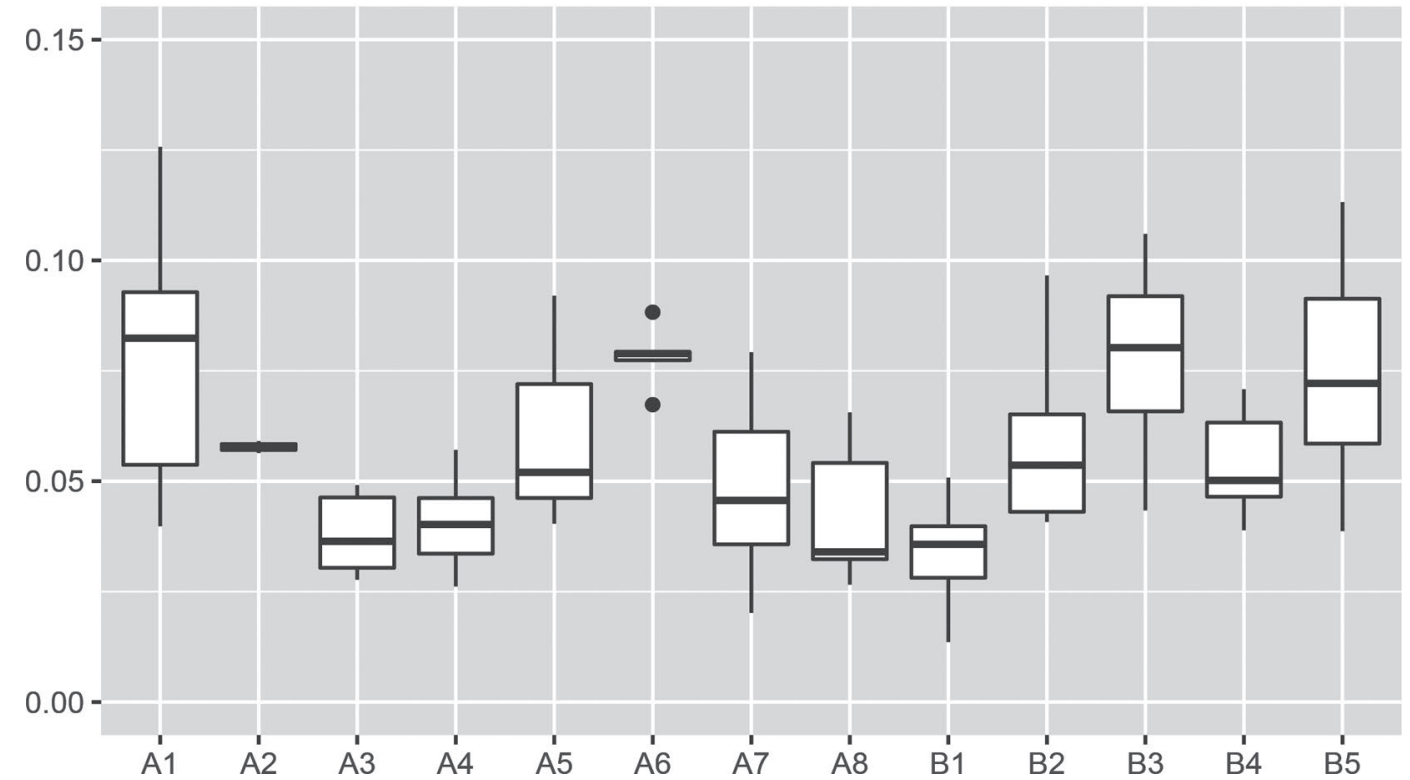

Figure 3. The (a) raw mean and (b) raw variance in pH series obtained from 93 animals from 13 farms. Horizontal lines denote medians; lower and upper hinges correspond to the first and third quantiles; whiskers extend to 1.5 times the interquartile range; and dots denote outlying points. 
Table 2. Sums of squares, degrees of freedom, and mean squares obtained from a linear model fitted to 616,945 $\mathrm{pH}$ observations from 93 animals on 13 farms $^{1}$

\begin{tabular}{lcrc}
\hline Model component & Sum of squares & df & Mean squares \\
\hline Farm & $8,422.9$ & 12 & 701.9 \\
Animal & $12,508.2$ & 80 & 156.4 \\
Daily sine wave & $3,435.8$ & 2 & $1,717.9$ \\
Milking sine wave & 567.7 & 2 & 283.9 \\
Farm $\times$ daily sine interaction & 573.3 & 24 & 23.9 \\
Farm $\times$ milking sine interaction & 539.2 & 24 & 22.5 \\
Animal $\times$ daily sine interaction & 578.6 & 160 & 3.6 \\
Animal $\times$ milking sine interaction & 325.6 & 160 & 2.0 \\
Residuals & $18,108.2$ & 616,480 & 0.0 \\
\hline
\end{tabular}

${ }^{1}$ Fixed effects of farm, animal, $\alpha_{1}$ and $\beta_{1}$ for the daily sine wave, and $\alpha_{2}$ and $\beta_{2}$ for the milking-frequency sine wave were fitted along with interactions, as indicated. The values for the corresponding $\alpha$ and $\beta$ effects have been combined to give an overall estimate for the relevant model component. Effects of farm and animal represent differences in mean $\mathrm{pH}$, effects of daily and milking sine waves reflect the importance of short-term temporal variation, and interactions indicate the variation in patterns between animals and farms.

pending on the animal (Figure 5a). Adding a sine wave to account for daily cyclical variability accounted for between 10 and $70 \%$ of the remaining variance, whereas adding a skew parameter $\gamma$ and a second sine wave corresponding to known milking times generally accounted for less than $10 \%$ of the remaining variance (Figure 5b-d). One interesting exception is that the milkingrelated sine wave accounted for between 7 and $35 \%$ of the remaining variance for all 24 animals on farm B1. A comparison of model fit using AIC indicated that the addition of the skew parameter $\gamma$ was supported for 82 of the 93 animals (Table 1 ), and the addition of the second sine wave was supported for 92 of the 93 animals. Models including the correct milking frequency were preferred for 61 of the $93(66 \%)$ animals, and models with the second sine wave adjusted to match the interval between milking events were preferred to models with regularly spaced time intervals for 72 out of the $93(77 \%)$ animals.

The daily pattern corresponding to the maximized likelihood of the models including a single sine wave with skew parameter (for ease of comparison between farms) is shown in Figure 6. The skew parameter (allowing different rates of increasing vs. decreasing $\mathrm{pH}$ ) varied between data series, with 46 showing a substantially faster increasing versus decreasing $\mathrm{pH}, 29$ showing a substantially faster decreasing versus increasing $\mathrm{pH}$, and the remaining 102 data series estimated as close to a standard sine wave. The amplitude was estimated between 0.1 and $0.2 \mathrm{pH}$ units for most animals. The plotted curves show a high degree of consistency for all animals in terms of the period of peak $\mathrm{pH}$ around 0600 to $0800 \mathrm{~h}$, with a corresponding trough at around $1800 \mathrm{~h}$ (Figure 6). The patterns appear to be more consistent within farm than between farms. For example, farm A6 shows a very similar signature for all animals that is almost unique to that farm. However, farm B1 shows 2 apparent subgroups of animals with patterns resembling those from farms B4 and A6, respectively, and there is also an individual pattern that does not closely resemble the majority at farms B3, A3, and A4. This suggests that despite the general consistency within a farm, individual-cow factors can result in unusual patterns in some animals relative to their herd mates. There is also a large amount of variation in the estimated mean $\mathrm{pH}$ values among animals, even within the same herd.

\section{Relating pH Abnormalities to Production Characteristics}

A total of $1,044 \mathrm{~d}$ of $\mathrm{pH}$ observations and model residuals were summarized for the 24 animals in herd B1. The patterns of mean residual and mean absolute residual indicated some similarities due to the correlation between the measurements (Supplemental Figure S1; https://doi.org/10.3168/jds.2017-12828). Most animals showed little qualitative evidence of strongly "abnormal" events, but deviations could be detected visually - for example, toward the end of the series in animals 5, 6, and 11 . These 3 events seem to be concurrent with abnormally high numbers of extreme $\mathrm{pH}$ observations (Supplemental Figure S2; https://doi .org/10.3168/jds.2017-12828), although the latter plot is more difficult to interpret.

There was a total of 1,043 observations of daily DMI and 970 observations of daily milk yield (after correcting for stage of lactation) that could be related to the $\mathrm{pH}$ observations in the 24 animals from herd B1. Results of the 2 multivariable models show that an increasing daily mean absolute residual had a significant $(P<0.001)$ and strongly negative effect on both production characteristics (Table 3). In contrast, neither the daily mean residual nor the number of extreme $\mathrm{pH}$ 

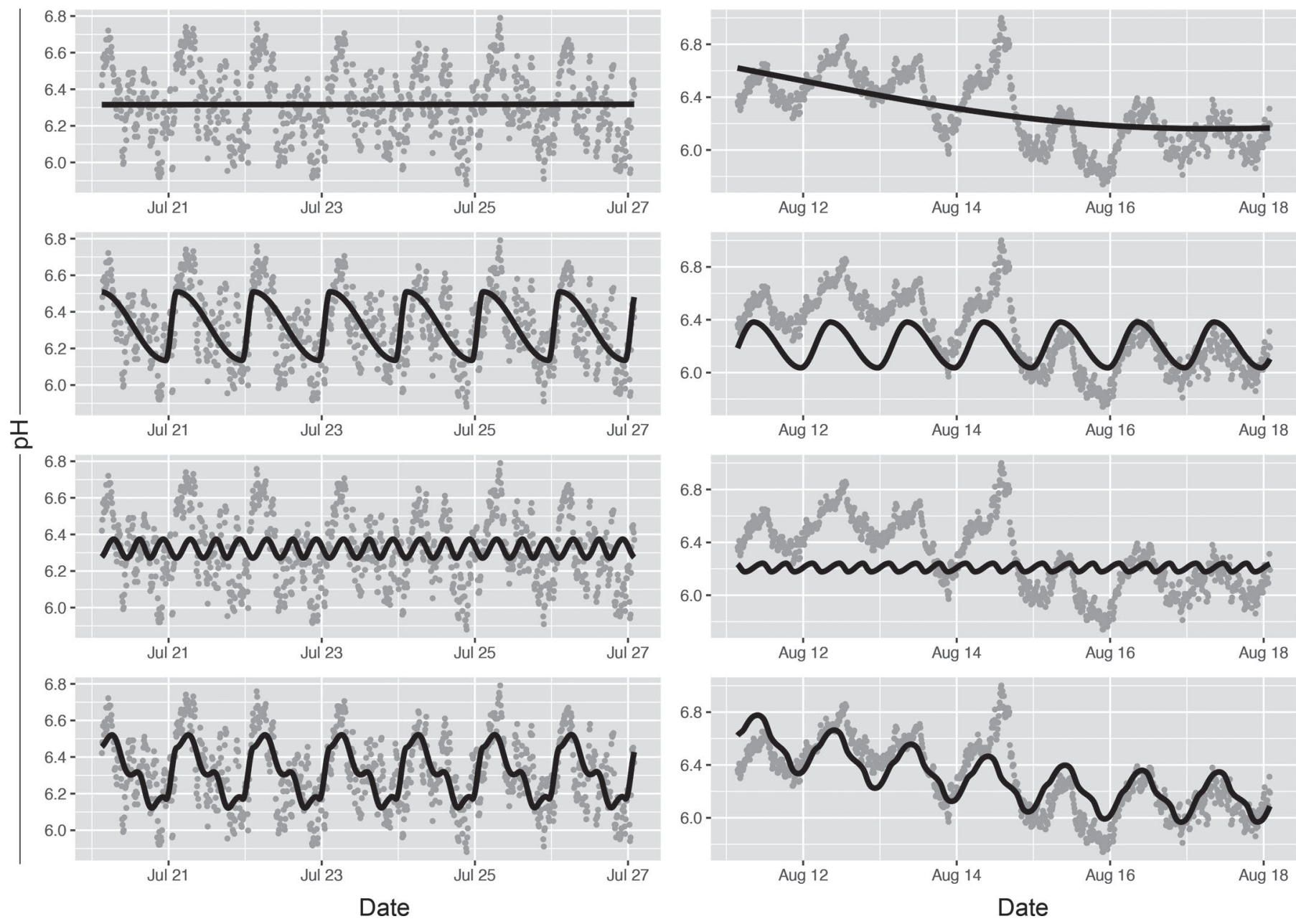

Figure 4. Illustration of the $\mathrm{pH}$ observations (gray dots) recorded over a 1-wk period for a randomly chosen animal from each of herd B1 (left) and A1 (right), overlaid with the predictions from the generalized additive model (top row), sine wave with daily frequency (second row), sine wave with milking frequency (third row), and combination of these 3 elements of the model (bottom row).

observations (either high or low $\mathrm{pH}$ ) had a significant effect on either milk yield or DMI. This indicates that deviations from a predictable daily rhythm (as represented by the mean absolute residual) are associated with decreased productivity, whereas neither changes in the daily average $\mathrm{pH}$ (as represented by the daily mean residual) nor extreme $\mathrm{pH}$ events are directly associated with productivity.

\section{DISCUSSION}

This article gives an overview of the characteristics of 93 continuously monitored $\mathrm{pH}$ data series obtained from animals from 13 different farms. No clinical or subclinical pathology (including digestive alterations) had been reported in these animals, so the results can be considered as a baseline of normal activity over a range of animals, although the possibility of undetected subclinical disease in some animals cannot be excluded. The importance of the observed daily fluctuation is striking, indicating that a consistent and substantial difference in $\mathrm{pH}$ should be expected between samples taken in the evening versus early morning. There were also stronger similarities between animals from the same farm than from different farms, presumably resulting from a range of dietary and management factors affecting the daily $\mathrm{pH}$ cycle of the rumen. However, there were also substantial differences between animals, particularly in terms of the average $\mathrm{pH}$ observed during the study, and the proportion of the overall variance that could be attributed to long-term predictable changes in $\mathrm{pH}$ and short-term cyclical behavior. There was also potentially biologically interesting variation in the peak difference in $\mathrm{pH}$ (amplitude) between animals, and particularly between farms. There was also substantial longer term temporal variation in some 
a)

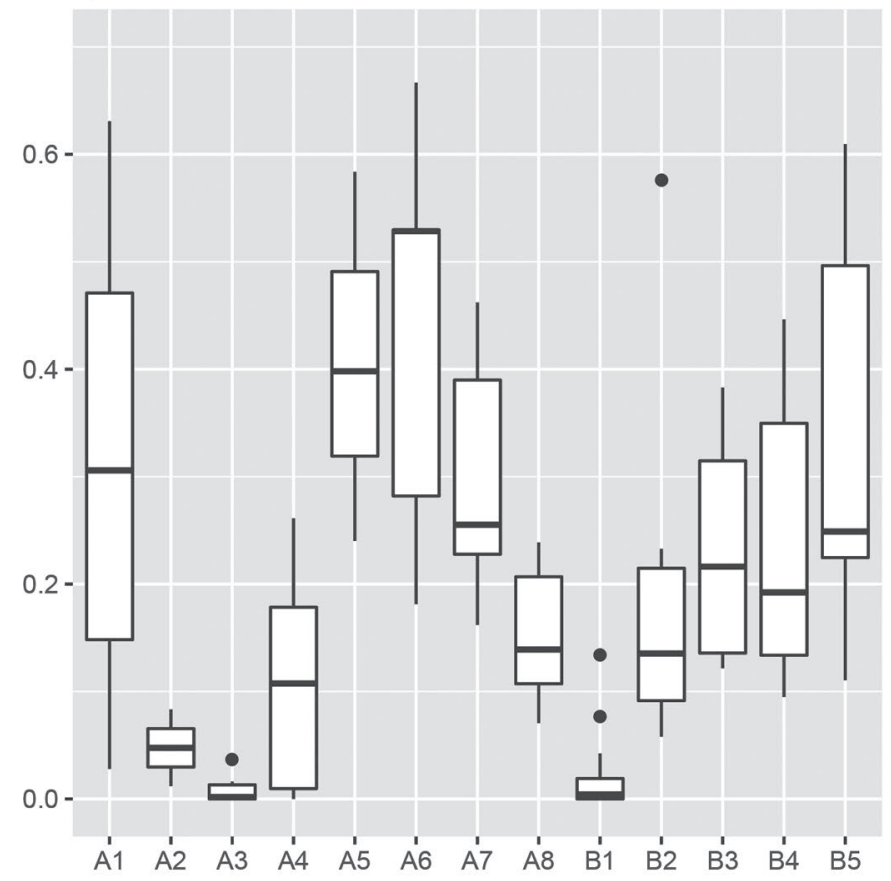

c)

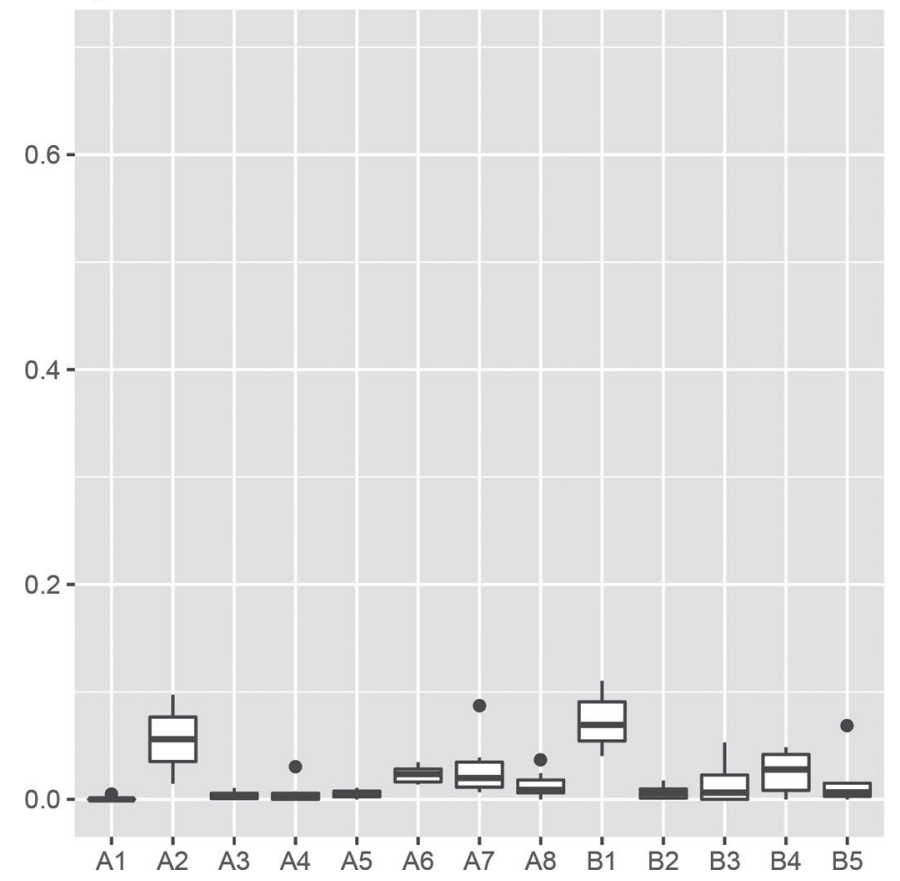

b)

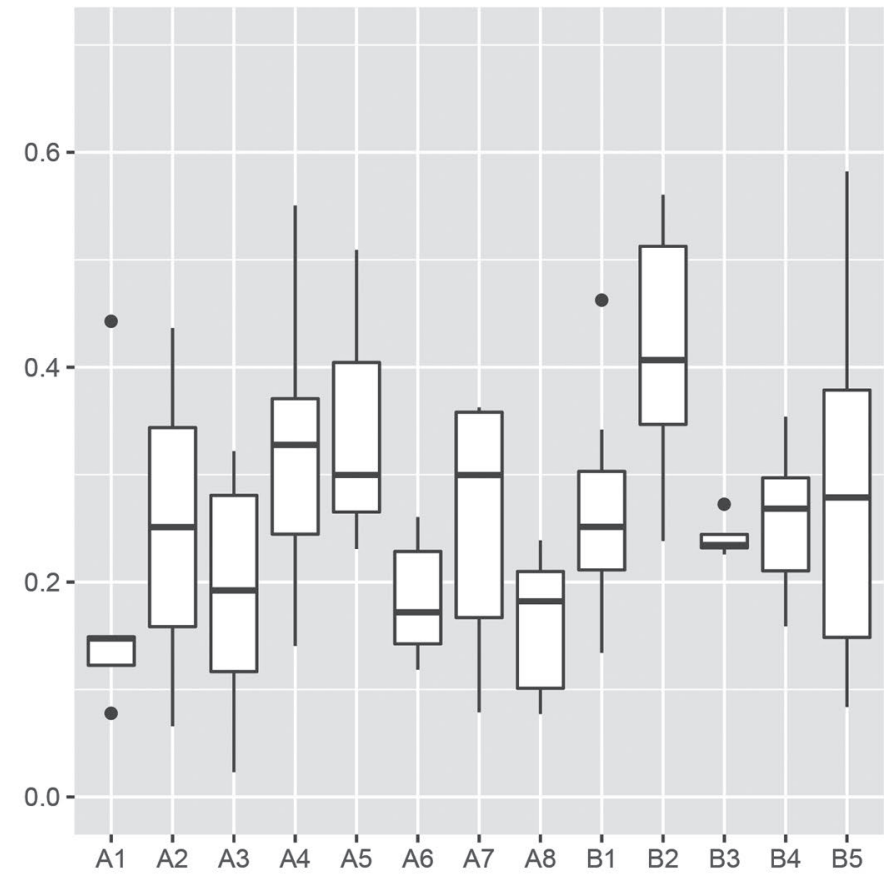

d)

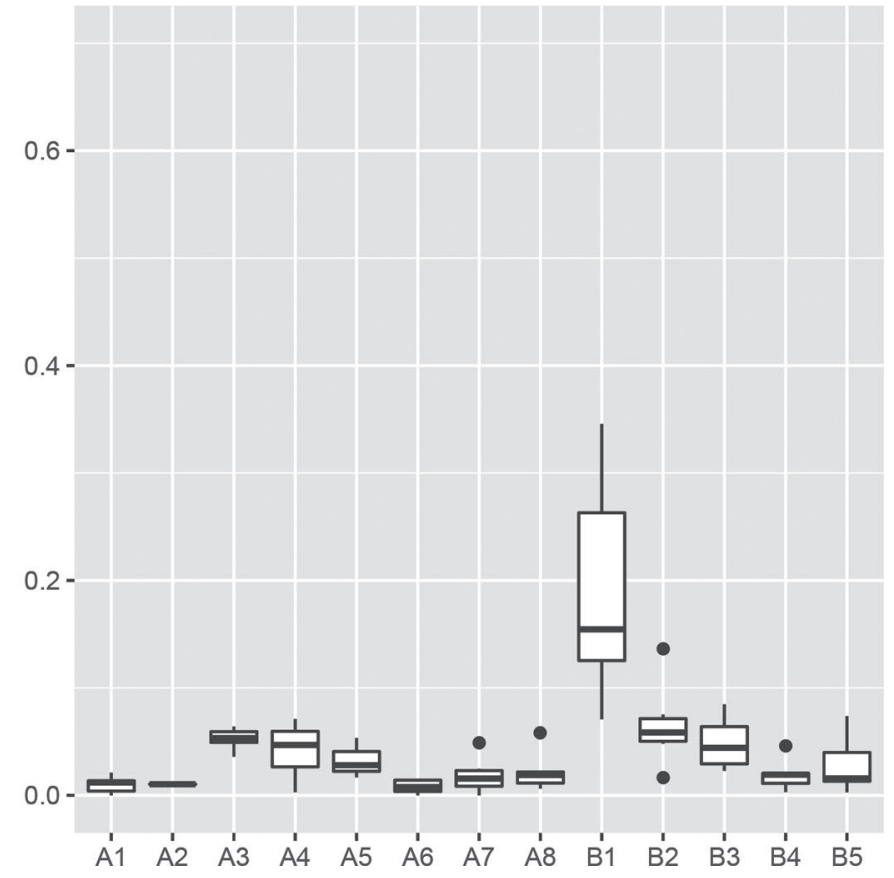

Figure 5. The (a) proportion of the total variance explained by a generalized additive model as well as the proportion of the remaining variance explained by adding (b) a single sine wave, (c) a skew parameter $(\gamma)$ to this sine wave, and then (d) a second sine wave to fit the known milking times on the farm. Horizontal lines denote medians; lower and upper hinges correspond to the first and third quantiles; whiskers extend to 1.5 times the interquartile range; and dots denote outlying points.

animals, which may reflect either gradual changes in $\mathrm{pH}$ function (due to, e.g., dietary factors) or $\mathrm{pH}$ drift in the sensors and necessitated the use of a GAM.
The model-fit comparison also allows some recommendations to be made in terms of modeling these data for other purposes. The addition of a sine wave 
to explain short-term variation in $\mathrm{pH}$ was strongly justified for all animals and was able to account for a substantial portion of the observed variation in $\mathrm{pH}$, suggesting that simple daily cyclic activity should be accounted for, particularly when comparing $\mathrm{pH}$ values observed at different times of the day. The addition of a second sine wave to account for management factors associated with the known frequency of milking was also supported, although this had less influence than the main sine wave. Results from the simpler linear model indicate relatively little variation in the parameters of these sine waves between animals on the same farm,
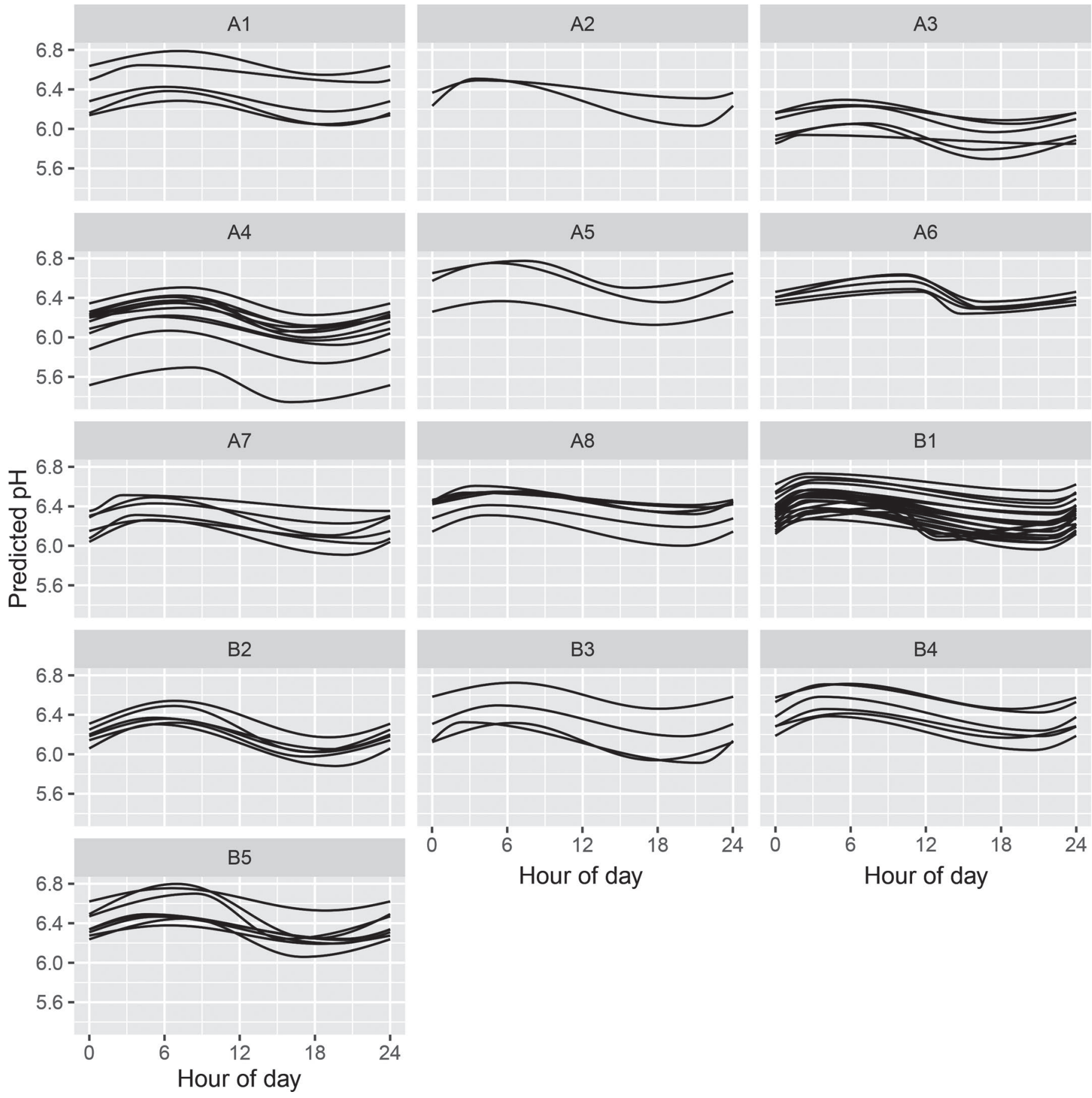

Figure 6. The fitted prediction from a single sine wave (including skew parameter) fitted to $\mathrm{pH}$ series obtained from 93 animals from 12 farms (longer term variability modeled by the generalized additive model is ignored). 
Table 3. Estimates obtained from 2 multivariable linear mixed models relating 4 different daily $\mathrm{pH}$ summary statistics to the daily corrected milk yield and daily DMI observed 2 d later ${ }^{1}$

\begin{tabular}{lcrrrrr}
\hline & \multicolumn{2}{c}{$\begin{array}{c}\text { Daily corrected } \\
\text { milk yield }\end{array}$} & & \multicolumn{2}{c}{ Daily DMI } \\
\cline { 2 - 3 } \cline { 5 - 6 } Item & Estimate & $P$-value & & Estimate & $P$-value \\
\hline Mean residual & -0.137 & 0.911 & & 0.019 & 0.988 \\
Mean absolute residual & -8.633 & $<0.001$ & & -8.698 & $<0.001$ \\
No. of observations below lowest 1\% & 0.041 & 0.056 & & 0.015 & 0.530 \\
No. of observations above highest 1\% & 0.003 & 0.877 & & 0.018 & 0.396 \\
\hline
\end{tabular}

${ }^{1}$ Summary statistics are mean residual $\mathrm{pH}$, mean absolute residual $\mathrm{pH}$, and number of $\mathrm{pH}$ observations below and above the most extreme $1 \%$ values for that animal.

which could justifiably be ignored in some situations. However, we recommend that differences in temporal variation between farms and inconsistent mean $\mathrm{pH}$ between animals on the same farm should always be taken into account. The addition of a skew parameter to allow $\mathrm{pH}$ to increase and decrease at different rates was also supported for the majority of animals but had a much smaller role in explaining the variance and requires the use of more complex nonlinear models. However, further research is warranted into the potential nutritional implications of this imbalance between the rates of increase and decrease in $\mathrm{pH}$. Finally, the fact that the "correct" frequency of milking was not consistently identified on most farms suggests that the improved fit of these models is partly attributable to the effects of fitting an additional harmonic frequency rather than a mechanistic effect related to milking. However, farm B1 consistently showed a substantially improved fit when including the correct frequency and for the timings linked to the estimated milking times rather than equally spaced throughout the day, suggesting that the sine wave reflects a real process on this farm. This could indicate a high degree of regularity in the relevant factors associated with the daily schedule of this farm (e.g., feeding times), which is far more likely to be the true cause of regular $\mathrm{pH}$ patterns than the milking process itself. Note that the most relevant management factors need not occur at the same time as milking, as each sine wave allows the peak and trough time to occur at any point during the cycle for each animal independently; only the frequency is fixed.

The focus of this article is to provide insight into the variation of qualitatively normal $\mathrm{pH}$ series between dairy cattle within and between farms. However, the ultimate goal of monitoring reticuloruminal $\mathrm{pH}$ is not to describe normality but rather to detect situations in which there is evidence for an abnormal pattern. Although they are not standard threshold $\mathrm{pH}$ values, the first and 99th percentiles for each animal were chosen to reflect the relatively unusual observations within $\mathrm{pH}$ series that did not contain observations generally considered to be extreme. There was no association between productivity and the frequency of these extreme $\mathrm{pH}$ observations. However, using the mean absolute residual between the fitted model and observed data over a daily time period, we demonstrated that it is possible to predict changes in the milk yield and DMI of the cow, even when based on data from clinically normal cows. Further work is required to refine this system to optimize the sensitivity to changes in $\mathrm{pH}$ variability and to develop models for real-time monitoring that are able to continuously adapt to the specific $\mathrm{pH}$ pattern of an individual animal based only on the previously observed values. A key goal of further research will be to investigate the predictive utility of reticuloruminal $\mathrm{pH}$ measurements based on past measurements, perhaps using a method such as a DLM (Madsen et al., 2005; Bono et al., 2012; Jensen et al., 2017). Using residuals from a DLM was previously shown to be useful for detecting specific production-relevant events such as mastitis in dairy cows (Jensen et al., 2016) and estrus in group-housed sows (Ostersen et al., 2010) as well as nonspecific events in animal production (Madsen and Kristensen, 2005; Cornou et al., 2008). Multivariate DLM also allows data series gathered from multiple different sources to be modeled simultaneously and the covariance between the different variables to be taken into account when forecasting expected observations (Jensen et al., 2015, 2016). It is therefore likely that using both reticuloruminal $\mathrm{pH}$ and feed intake data with a combined model would result in improved performance in detecting undesirable events when compared with $\mathrm{pH}$ data alone.

\section{CONCLUSIONS}

Continuously monitored reticuloruminal $\mathrm{pH}$ data show a strong and predictable short-term pattern that is well described by a simple sine wave with a frequency of 1 cycle per day. More complex models, including an additional sine wave and a skew parameter, give incrementally smaller additional benefits but can be used 
to demonstrate differences between animals. Deviations from the expected daily cyclical variation were significantly associated with reduced production, but there was no apparent effect of extreme $\mathrm{pH}$ observations on productivity. We therefore conclude that future efforts to describe continuously monitored $\mathrm{pH}$ data should be based on deviations from an expected predictable rhythm rather than observed measurements below some arbitrarily defined $\mathrm{pH}$ threshold.

\section{ACKNOWLEDGMENTS}

The authors gratefully acknowledge Sarah Layhe (Vaetta Editing, Copenhagen, Denmark) for assistance with proofreading and formatting of the manuscript and the 2 anonymous reviewers for their excellent suggestions, which have greatly improved the manuscript. Dan Jensen was financially supported by the Danish Council for Strategic Research (Copenhagen, Denmark; the PigIT project, grant no. 11-116191).

\section{REFERENCES}

Akaike, H. 1973. Information theory as an extension of the maximum likelihood principle. Pages 267-281 in Proc. Second International Symposium on Information Theory. B. N. Petrov and F. Csaki, ed. Akademiai Kiado, Budapest, Hungary.

Ali, T. E., and L. R. Schaeffer. 1987. Accounting for covariances among test day milk yields in dairy cows. Can. J. Anim. Sci. 67:637-644. https://doi.org/10.4141/cjas87-067.

Bates, D., M. Maechler, B. Bolker, and S. Walker. 2014. lme4: Linear mixed-effects models using Eigen and S4. R package version 1.1-7. Accessed May 1, 2015. http://cran.r-project.org/package=lme4.

Bono, C., C. Cornou, and A. R. Kristensen. 2012. Dynamic production monitoring in pig herds I: Modeling and monitoring litter size at herd and sow level. Livest. Sci. 149:289-300. https://doi.org/10 .1016/j.livsci.2012.07.023.

Buttchereit, N., E. Stamer, W. Junge, and G. Thaller. 2010. Evaluation of five lactation curve models fitted for fat:protein ratio of milk and daily energy balance. J. Dairy Sci. 93:1702-1712. https:// doi.org/10.3168/jds.2009-2198.

Cole, J. B., D. J. Null, and P. M. Vanraden. 2009. Best prediction of yields for long lactations. J. Dairy Sci. 92:1796-1810. https://doi .org/10.3168/jds.2007-0976.

Cornou, C., J. Vinther, and A. R. Kristensen. 2008. Automatic detection of oestrus and health disorders using data from electronic sow feeders. Livest. Sci. 118:262-271. https://doi.org/10.1016/j.livsci 2008.02 .004

Dirksen, G., and M. C. Smith. 1987. Acquisition and analysis of bovine rumen fluid. Bov. Pract. 22:108-116.

Elzhov, T. V, K.M. Mullen, A.-N. Spiess, and B. Bolker. 2013. minpack.lm: $\mathrm{R}$ interface to the Levenberg-Marquardt nonlinear leastsquares algorithm found in MINPACK, plus support for bounds.
$\mathrm{R}$ package version 1.1-8. Accessed May 1, 2015. https://cran.r -project.org/package=minpack.lm.

Garrett, E. F., M. N. Pereira, K. V. Nordlund, L. E. Armentano, W. J. Goodger, and G. R. Oetzel. 1999. Diagnostic methods for the detection of subacute ruminal acidosis in dairy cows. J. Dairy Sci. 82:1170-1178. https://doi.org/10.3168/jds.S0022-0302(99)75340 $-3$.

Jensen, D. B., C. Cornou, N. Toft, and A. R. Kristensen. 2015. A multi-dimensional dynamic linear model for monitoring slaughter pig production. Pages 503-512 in 7th European Conference on Precision Livestock Farming. Accessed Oct. 6, 2017. http://users .unimi.it/ecplf2015/.

Jensen, D. B., H. Hogeveen, and A. De Vries. 2016. Bayesian integration of sensor information and a multivariate dynamic linear model for prediction of dairy cow mastitis. J. Dairy Sci. 99:7344-7361. https://doi.org/10.3168/jds.2015-10060.

Jensen, D. B., N. Toft, and A. R. Kristensen. 2017. A multivariate dynamic linear model for early warnings of diarrhea and pen fouling in slaughter pigs. Comput. Electron. Agric. 135:51-62. https://doi .org/10.1016/j.compag.2016.12.018.

Khafipour, E., J. C. Plaizier, P. C. Aikman, and D. O. Krause. 2011 Population structure of rumen Escherichia coli associated with subacute ruminal acidosis (SARA) in dairy cattle. J. Dairy Sci. 94:351-360. https://doi.org/10.3168/jds.2010-3435.

Maatje, K., P. J. M. Huijsmans, W. Rossing, and P. H. Hogewerf. 1992. The efficacy of in-line measurement of quarter milk electrical conductivity, milk yield and milk temperature for the detection of clinical and subclinical mastitis. Livest. Prod. Sci. 30:239-249. https://doi.org/10.1016/S0301-6226(06)80013-8.

Madsen, T. N., S. Andersen, and A. R. Kristensen. 2005. Modelling the drinking patterns of young pigs using a state space model. Comput. Electron. Agric. 48:39-62. https://doi.org/10.1016/j .compag.2005.01.001.

Madsen, T. N., and A. R. Kristensen. 2005. A model for monitoring the condition of young pigs by their drinking behaviour. Comput. Electron. Agric. 48:138-154. https://doi.org/10.1016/j.compag .2005.02.014

Nordlund, K. V., and E. F. Garrett. 1997. Rumenocentesis: A technique for collecting rumen fluid for the diagnosis of subacute rumen acidosis in dairy herds. Proc. Univ. Sydney Post Grad. Found. Vet. Sci. 290:195-200.

Nordlund, K. V., E. F. Garrett, and G. R. Oetzel. 1995. Herd-based rumenocentesis: A clinical approach to the diagnosis of subacute rumen acidosis. Compend. Contin. Educ. Pract. Vet. 17(8 Suppl.):S48-S56.

Ostersen, T., C. Cornou, and A. R. Kristensen. 2010. Detecting oestrus by monitoring sows' visits to a boar. Comput. Electron. Agric. 74:51-58. https://doi.org/10.1016/j.compag.2010.06.003.

Plaizier, J. C., D. O. Krause, G. N. Gozho, and B. W. McBride. 2008. Subacute ruminal acidosis in dairy cows: The physiological causes, incidence and consequences. Vet. J. 176:21-31. https://doi.org/10 $.1016 /$ j.tvjl.2007.12.016.

R Core Team. 2016. R: A language and environment for statistical computing. R Foundation for Statistical Computing, Vienna, Austria. Version 3.2.4. Accessed Apr. 1, 2016. https://www.r-project .org/.

Wood, P. D. 1967. Algebraic model of the lactation curve in cattle. Nature 216:164-165.

Wood, S. N. 2011. Fast stable restricted maximum likelihood and marginal likelihood estimation of semiparametric generalized linear models. J. R Stat. Soc. 73:3-36. 